\title{
Investigation of the Sensitivity and Specificity of Laboratory Tests Used in Differential Diagnosis of Childhood Brucellosis
}

\author{
Halil Kazanasmaz $^{1}$, Süleyman Geter ${ }^{2}$ \\ 1. Pediatrics, Harran University Medical School, Sanliurfa, TUR 2. Pediatrics, Sanliurfa Training and Research Hospital, \\ Sanliurfa, TUR
}

Corresponding author: Halil Kazanasmaz, kazanasmazhalil@yahoo.com

\section{Abstract \\ Objectives}

Childhood brucellosis is a common public health problem in developing countries. The diagnosis of brucellosis based on nonspecific symptoms is an ongoing problem for physicians, especially in endemic areas. In this study, it is aimed to discuss the efficacy of frequently used test methods in the differential diagnosis of brucellosis.

\section{Methods}

The records of 332 patients admitted to pediatric clinic on suspicion of brucellosis were retrospectively analyzed. Patients with brucellosis were included in the positive group $(\mathrm{n}=262)$ and those without brucellosis were included in the negative group $(n=70)$.

\section{Results}

As a result of biochemical analysis of the cases, median alanine aminotransferase (ALT), aspartate aminotransferase (AST), gamma-glutamyl transferase (GGT), erythrocyte sedimentation rate (ESR), and C reactive protein (CRP) values were significantly higher in the positive group than that in the negative group $(\mathrm{p}<0.05)$. There was no significant difference between median white blood cell, neutrophil, lymphocyte, neutrophil to lymphocyte ratio, hemoglobin, and platelet values between groups ( $\mathrm{p}>0.05)$. Receiver operating curves were plotted to compare predictive values of CRP (area under curve (AUC): 0.635 , $\mathrm{p}=0.001$ ), ESR(AUC:0.701, $\mathrm{p}<0.001$ ), AST(AUC: 0.595, $\mathrm{p}=0.015$ ), ALT(AUC:0.604, $\mathrm{p}=0.007$ ), and GGT(AUC:0.593, $\mathrm{p}=0.016$ ) in 332 patients with suspected brucellosis.

\section{Conclusions}

Increased levels of AST, ALT, GGT, CRP, and ESR may have a complementary role in the differential diagnosis of childhood brucellosis. However, all of these markers should be evaluated with clinical findings due to

Received 01/17/2020

Review began 01/20/2020 Review ended 01/21/2020 Published 01/23/2020

\section{(c) Copyright 2020}

Kazanasmaz et al. This is an open access article distributed under the terms of the Creative Commons Attribution License CC-BY 3.0., which permits unrestricted use, distribution, and reproduction in any medium, provided the original author and source are credited.
Categories: Pediatrics, Infectious Disease, Epidemiology/Public Health

Keywords: brucellosis, c reactive protein, differential diagnoses, erythrocyte sedimentation rate

\section{Introduction}

Childhood brucellosis is a zoonotic disease transmitted to humans through infected animals [1]. Brucellosis confronts public health currently as a widespread problem all over the world and it is especially common in the Middle East including Turkey [1-2]. The incidence of this zoonotic disease in Turkey is around 23-25 per 100,000 annually [1,3]. Brucellosis is frequent in the rural areas of Turkey, especially in Central and Southeastern Anatolia, where animal husbandry is common. Among the causative strains, Brucella melitensis is the most commonly isolated strain $[1,3]$.

Animals and animal products are the main sources to cause brucellosis in humans. Contamination occurs via the consumption of unpasteurized dairy products and/or raw meat and through direct contact with infected animals in the presence of erosions on the skin and mucosa. Farmers, veterinarians, abattoir workers, and workers in animal husbandry or people in contact with animals are at risk of infection. If those animals are kept close to the living environment of humans, animal exposure will potentially be inevitable and the families of these people will be at high risk, too [4]. Fever and monoarthritis, often involving the hip and/or knee joint are common clinical findings in brucellosis; however, no disease-specific pathognomonic signs and symptoms exist [5-7]. Although rarely, brucellosis can be associated with a variety of clinical findings; including acute renal failure, endocarditis, splenic abscess, spondylitis, and encephalitis $[4,8-9]$. Laboratory tests can often yield normal inflammatory biomarker levels [5]. 
The definitive diagnosis of brucellosis is made by isolating the microorganism from the blood or various tissue samples (such as the bone marrow, synovial fluid, etc.) or by identifying the causative agent based on the results of molecular tests like polymerase chain reaction (PCR) [10]. Besides the most commonly used diagnostic clinical laboratory test methods including Rose Bengal plate agglutination (RBPT), standard tube agglutination test (SAT), coombs antiglobulin test, and enzyme-linked immunosorbent assay; blood culture is recognized as the gold standard test [11-12]. Diagnosing brucellosis is challenging because the diagnostic methods have advantages and disadvantages over each other; no clinical pathognomonic findings have been described yet, and the levels of inflammatory biomarkers can be normal [5,12-13].

This study aimed to discuss the efficacy of commonly used test methods in the diagnosis of brucellosis in light of the information in the literature and to demonstrate the clinical characteristics in pediatric patients from Şanlıurfa province, where brucellosis is endemic.

\section{Materials And Methods}

Patients, who were admitted to the pediatric clinics of two hospitals in Şanlıurfa province due to clinical suspicion of brucellosis in the period from January 2018 to December 2018, were included in the study. Before the study, written informed consent was obtained from the parents of the patients who participated in this study. This study conformed to the principles of the 2008 Declaration of Helsinki and was approved by the local ethics committee in Turkey. Immunological diagnosis of brucellosis was made using serological methods. All patients underwent RBPT and SAT analyses simultaneously. RBPT positive patients were included in the study. Patients were accepted positive for brucellosis when both titers were 1/160 or more in two SAT tests performed consecutively at two-week intervals besides a positive RBPT result. Patients were excluded when the first titer was positive but the second titer was negative. Patients were accepted negative despite RBPT positivity when SAT negative patients $(<1 / 160)$ had negative SAT results $(1 / 160)$ again in the following second week; additionally to negative blood culture results and no clinical suspicion of brucellosis.

Collection of Blood Samples

Blood culture samples were collected from peripheral antecubital veins appropriately. A total of $2 \mathrm{ml}$ of the blood sample was placed in one aerobic and one anaerobic blood culture tubes under sterile conditions. The BD BACTEC ${ }^{\text {тм }}$ FX40 blood culture system (Becton Dickinson Diagnostics, Sparks, MD, USA) was used for isolating microorganisms from the blood. A blood sample volume of $2 \mathrm{~mL}$ was placed in gel-containing tubes for biochemical analyses and tube agglutination test, and into K2 EDTA tubes (potassium-2 ethylene diamine tetraacetic acid) for laboratory tests, absolute blood count, and erythrocyte sedimentation rate (ESR) measurements according to the manufacturer's instructions. ESR measurements were obtained with an automatic Vision (Shenzhen YHLO Biotech, China) device. Complete blood counts were calculated with an automatic blood cell counting device (Abbot Celldyn 3500 Ill, USA). As a result of the analysis, white blood cell (WBC), neutrophil (NEU), lymphocyte (LYM), hemoglobin ( $\mathrm{Hb}$ ), and platelet (PLT) values were obtained. Neutrophil to lymphocyte ratio (NLR) was obtained by dividing the absolute NEU count by the absolute LYM count. Biochemical analysis results were obtained using a spectrophotometric chemical analyser Architect C16000 (Abbott Diagnostics, Abbott Park, USA). As a result of the analysis, alanine aminotransferase (ALT), aspartate aminotransferase (AST), C reactive protein (CRP), and gamma-glutamyl transferase (GGT) values were obtained.

\section{Rose Bengal Plate Agglutination Test}

The standard method for RBPT was performed on a glass plate [14]. Blood serum samples of 30 ul antigen and $30 \mathrm{ul}$ serum were placed onto a glass slide and mixed well at room temperature. Any degree of agglutination visible to a naked eye within four minutes was considered positive.

\section{Standard Tube Agglutination Test}

The serum in the blood samples was tested in glass tubes at the following dilutions of $1 / 40,1 / 80,1 / 160$, $1 / 320,1 / 640,1 / 1280$, and $1 / 2560$ using the Cromatest (Linear Chemicals Montgat, Spain) kit according to the manufacturer's instructions. Patients with a titer of $1 / 160$ and above were considered positive.

Analysis of the Data

Statistical analyses were performed using the SPSS 24.0 version (SPSS Inc., Chicago, IL) package program. Descriptive statistics were summarized as median, minimum, and maximum values. The suitability of the variables to the normal distribution was investigated using visual (histogram and probability charts) and analytical methods (Kolmogorov-Smirnov, Shapiro-Wilk tests). Mann-Whitney U test was used to analyze the data of the groups that were not homogeneous and had a different number of cases. Categorical variables were analyzed using Pearson chi-square test. Specificity and sensitivity analyses were performed using receiver operating curve (ROC) analysis method. In the ROC analysis, the area under curve (AUC) values were studied. The comparisons were considered to be statistically significant when the probability (p) value in the 


\section{Cureus}

study analysis was less than 0.05 .

\section{Results}

This study included 332 patients, who were referred to pediatric clinics with suspicion of brucellosis. While $78.9 \%$ of the study participants were positive, $21.1 \%$ were negative (Table 1 ). In the positive group, $52.3 \%$ were males and $47.7 \%$ were females while in the negative group, $54.3 \%$ were males and $47.3 \%$ were females. The comparison of the two groups with the Pearson chi-square test revealed no significant differences in gender distribution $(\mathrm{p}=0.766)$. The mean age of the patients in the positive group was $10.48 \pm 4.48$ (min$\max , 1-18$ ) years, whereas the mean age of the patients in the negative group was $9.69 \pm 4.39$ (min-max, 1-18) years. The Mann-Whitney $U$ test did not reveal any differences in the age distribution between the two groups $(\mathrm{p}=0.165)$.

\begin{tabular}{|c|c|c|c|}
\hline & Positive group ( $n=262)$ & Negative group $(n=70)$ & P value \\
\hline Age/Year median(min-max) & $11(1-18)$ & $9(1-18)$ & $0.165^{\mathrm{a}}$ \\
\hline Gender M/F n(\%) & $137(52.3) / 125(47.7)$ & $38(54.3) / 32(45.7)$ & $0.766^{b}$ \\
\hline \multicolumn{4}{|l|}{ Seasons n(\%) } \\
\hline Spring & 34(13) & 16(22.9) & \multirow{4}{*}{$0.006^{b}$} \\
\hline Summer & $94(35.9)$ & 13(18.6) & \\
\hline Autumn & 88(33.6) & $21(30)$ & \\
\hline Winter & $46(1 / .6)$ & $20(28.6)$ & \\
\hline Rural and Farming / Urban n(\%) & 216(82.4)/46(17.6) & $41(58.6) / 29(41.4)$ & $<0.001^{b}$ \\
\hline Case in family Positive/Negative $n(\%)$ & 64(24.4)/198(75.6) & $5(7.1) / 65(92.9)$ & $0.002^{\mathrm{b}}$ \\
\hline Unpasteurized milk consumption Positive/Negative $n(\%)$ & 165(63)/97(37) & $27(38.6) / 43(61.4)$ & $<0.001^{b}$ \\
\hline
\end{tabular}

\section{TABLE 1: Sociodemographic and clinical characteristics of cases}

a:Mann-Whitney U test; ${ }^{\text {b: }}$ Pearson chi-square test

Patients with positive test results (35.9\%) were found out to present to the hospital mostly in summer, while autumn was the most common admission season (30\%) in the negative group. A significant difference in the seasonal distribution of the cases was found between the two groups based on the Pearson chi-square test results $(\mathrm{p}=0.006$ ). While $24.4 \%$ of the positive group had brucellosis in the family history, $7.1 \%$ of the negative group had brucellosis in their family history. A significant difference was found in the frequency of family history of brucellosis between the groups $(\mathrm{p}=0.002)$ (Table 1$)$. Of the study patients, $63 \%$ of the positive group and $38.6 \%$ of the negative group reported consumption of unpasteurized milk and dairy products in medical history. The frequency of consumption of unpasteurized milk and dairy products were significantly different between the groups ( $\mathrm{p}<0.001$ ). While $82.4 \%$ of the cases in the positive group lived in rural areas and engaged in animal husbandry, this rate was $58.6 \%$ in the negative group. The frequency of study participants living in rural areas and working in animal husbandry was significantly different between the two groups $(\mathrm{p}<0.001)$.

In the positive group with the brucellosis diagnosis; arthralgia (45.8\%), fever $\left(\geqslant 38.5 \mathrm{C}^{0}\right)(28.6 \%)$, and malaise and fatigue (11.5\%) were the most common complaints at the time of presentation to the hospital (Table 2). In the positive group, the physical examination findings were nonspecific in $50.4 \%$ of the cases, while $32.4 \%$ of the patients had arthritis and $11.5 \%$ had hepatosplenomegaly. Of the patients in the positive group, $13.4 \%$ were hospitalized and $86.6 \%$ of them were treated as outpatients. Brucellosis was isolated in the blood cultures in only $4.2 \%$ of the positive group. 


\section{Cureus}

( $n=262)$

Complaints $n(\%)$

Arthralgia

Fever $\left(\geq 38.5 C^{0}\right)$

Malaise and fatigue

Abdominal pain

Night sweating

Weight loss

Physical examination $\mathrm{n}(\%)$

Arthritis

Hepatomegaly

Splenomegaly

Lymphadenopathy

Nonspecific rash

No findings

Treatment n(\%) Hospitalization / Outpatient

STA titer $\mathrm{n}(\%)$

$1: 160$

$1: 320$

$1: 640$

$1: 1280$

$1: 2560$
$70(26.7)$

Number of cases, $\mathrm{n} / \%$

120(45.8)

75 (28.6)

$30(11.5)$

19(7.3)

9(3.4)

9(3.4)

17(6.5)

13(5)

11(4.2)

4(1.5)

132(50.4)

$35(13.4) / 227(86.6)$

57(21.8)

114(43.5)

17(6.5)

4(1.5)

\section{TABLE 2: Clinical features of positive cases with brucellosis}

STA: Standard tube agglutination test.

Laboratory analysis results of the positive and negative groups were compared with the Mann-Whitney U test. There were no significant differences between the groups in the median WBC, LYM, NEU, NLR, Hb, and PLT counts revealed by the hematological analysis ( $p>0.05)$ (Table 3). The analysis of the biochemical test results revealed that the median AST, ALT, GTT, ESR, and CRP values were significantly higher in the positive group compared to the negative group $(\mathrm{p}<0.05)$ (Table 3). 


\section{Cureus}

\begin{tabular}{|c|c|c|c|}
\hline & Positive group $(n=262)$ & Negative group $(n=70)$ & P value ${ }^{a}$ \\
\hline 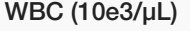 & 8.3(1.85-70.37) & $8.5(4.91-19.2)$ & 0.603 \\
\hline NEU (10e3/uL) & $3.6(0.6-14.78)$ & $4(1.10-12.98)$ & 0.129 \\
\hline LYM (10e3/uL) & $4(0.97-11.9)$ & $4(1.7-11.9)$ & 0.860 \\
\hline NLR & $0.99(0.22-6.35)$ & $1.08(0.15-4.45)$ & 0.353 \\
\hline $\mathrm{Hb}(\mathrm{g} / \mathrm{dL})$ & $13(9.5-16.4)$ & 13.4(10.5-15) & 0.406 \\
\hline 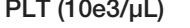 & $303.65(13.81-651)$ & 325(171-452.36) & 0.469 \\
\hline ALT (U/L) & $24.5(8-547)$ & $21(6-85)$ & 0.007 \\
\hline AST (U/L) & $32(7-569)$ & 28(12-69) & 0.015 \\
\hline GGT (U/L) & 14(8-128) & $11(9-51)$ & 0.016 \\
\hline CRP (mg/dL) & $0.3(0-15.94)$ & $0.18(0-7.14)$ & $<0.001$ \\
\hline $\operatorname{ESR}(\mathrm{mm} / \mathrm{h})$ & $21(5-116)$ & 13(5-88) & $<0.00$ \\
\hline
\end{tabular}

\section{TABLE 3: Comparison of laboratory analysis of positive and negative groups}

a: Mann-Whitney U test; WBC: White blood cell; NEU: Neutrophil; LYM: Lymphocyte; Hb:Hemoglobin; PLT: Platelets; GGT: Gamma-glutamyl transferase; ALT: Alanine aminotransferase; AST: Aspartate aminotransferase; CRP: C reactive protein; ESR: Erythrocyte sedimentation rate; NLR: Neutrophil to lymphocyte ratio

ROC curves were drawn to compare the predictive values of the laboratory test results of the 332 subjects included in the study (Figure 1). The analysis revealed that AUC for AST was 0.595 and the p-value was 0.015, AUC for ALT was 0.604 and the p-value was 0.007 , AUC for GGT was 0.593 and the p-value was 0.016 , AUC for CRP was 0.635 and the p-value was 0.001, and AUC for ESR was 0.701 and the p-value was $<0.001$ (Table 4).

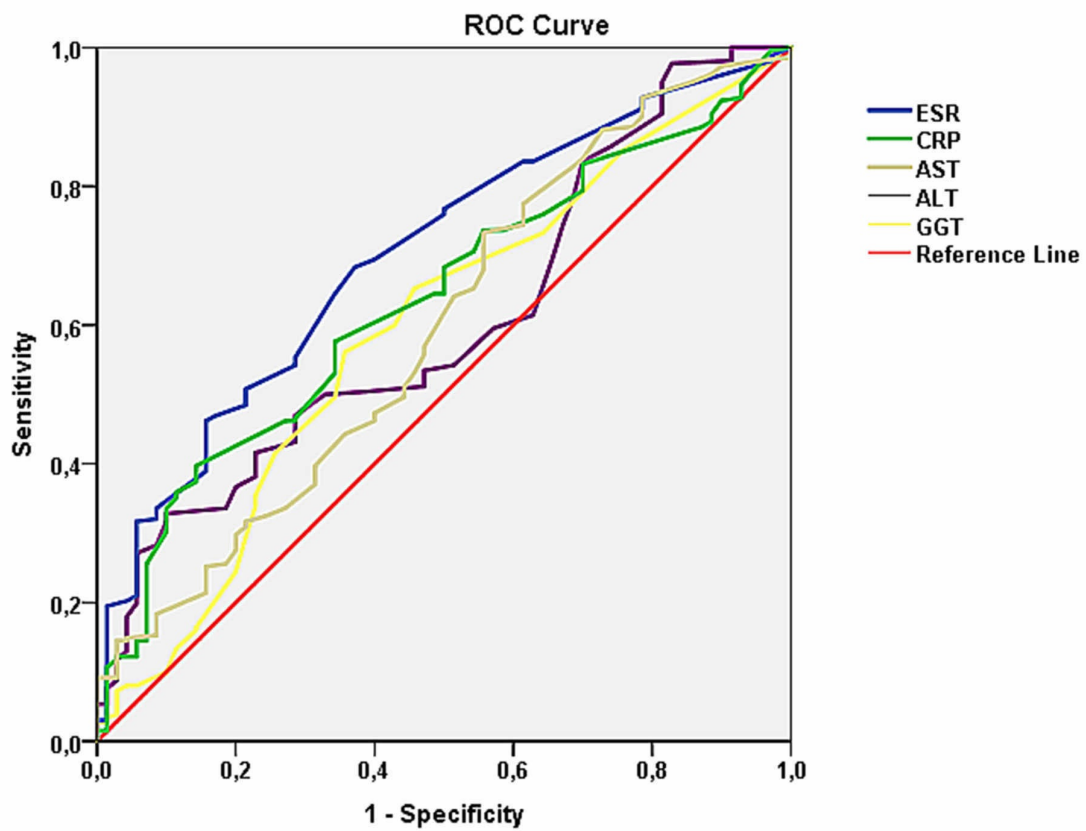

FIGURE 1: Comparison of specificity and sensitivity with receiver operating characteristic graph

ROC: Receiver Operating Curve; GGT: gamma-glutamyl transferase; ALT: alanine aminotransferase; AST: aspartate aminotransferase; CRP: C reactive protein; ESR: erythrocyte sedimentation rate 


\begin{tabular}{|c|c|c|c|c|c|}
\hline & Values & AUC & Sensitivity & Specificity & P value \\
\hline ALT (U/L) & 21.5 & 0.604 & 53.4 & 52.9 & 0.007 \\
\hline AST (U/L) & 31.5 & 0.595 & 53.1 & 54.3 & 0.015 \\
\hline GGT (U/L) & 12.5 & 0.593 & 59.9 & 57.1 & 0.016 \\
\hline CRP (mg/dL) & 0.21 & 0.635 & 57.6 & 65.7 & 0.001 \\
\hline ESR (mm/h) & 15.5 & 0.701 & 68.3 & 62.9 & $<0.001$ \\
\hline WBC $(10 \mathrm{e} 3 / \mu \mathrm{L})$ & - & 0.480 & - & - & 0.604 \\
\hline 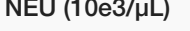 & - & 0.441 & - & - & 0.130 \\
\hline 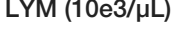 & - & 0.507 & - & - & 0.860 \\
\hline NLR & - & 0.464 & - & - & 0.353 \\
\hline 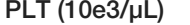 & - & 0.472 & - & - & 0.469 \\
\hline
\end{tabular}

\section{TABLE 4: ROC analysis results for AST, ALT, GGT, ESR, and CRP measurements}

ROC: Receiver Operating Curve; AUC: Area under the curve; GGT: Gamma-glutamyl transferase; ALT: Alanine aminotransferase; AST: Aspartate aminotransferase; CRP: C reactive protein; ESR: Erythrocyte sedimentation rate; WBC: White blood cells; NEU: neutrophil; LYM: lymphocyte; NLR: Neutrophil to lymphocyte ratio; PLT: platelet.

\section{Discussion}

Brucellosis is a systemic infectious disease that can be manifested by various clinical pictures [15-20]. Although brucellosis is well-controlled in developed countries, the disease remains to be a major health problem in developing countries including Iran and Turkey [16,20]. Brucellosis is especially seen in the Eastern and Southeastern Anatolia regions of Turkey, where livestock farming is common [15]. The disease is more common in boys at the age range from 9 to 11 years born to families engaged in animal husbandry in rural areas, where consumption of unpasteurized milk and dairy products is widespread [16,19]. In our study, $52.3 \%$ of the patients positive for brucellosis were males at a mean age of $10.48 \pm 4.48$ years. Of the brucellosis patients, $63 \%$ consumed unpasteurized milk and dairy products and $82.4 \%$ of them lived in the countryside with their families engaged in animal husbandry. Diagnosing brucellosis based on nonspecific disease symptoms is still an issue for physicians, especially working in endemic areas. While fever, night sweats, joint pain, malaise, and fatigue are nonspecific symptoms of brucellosis; arthralgia, hepatomegaly, and myalgia are the most common complaints $[11,16,20]$. Similarly, in our study, the main complaints were arthralgia, fever, malaise, and fatigue.

Clinical manifestations of brucellosis may range from nonspecific symptoms such as long-term fever, fatigue, and malaise to local organ involvement such as arthritis and neurobrucellosis [21]. Therefore, many studies have been carried out to determine the laboratory parameters, which will support clinical findings. Anemia, leukopenia, and especially in endemic areas thrombocytopenia can be detected in the complete blood count in patients with brucellosis [19-22]. Additionally, it has been reported that increased levels of inflammatory markers such as CRP, ESR, and liver enzymes such as AST, ALT, and GTT in biochemical tests can support the diagnosis [23-27]. A study by Bozdemir et al. reported that increased NLR values could be used as an indicator of inflammation in childhood brucellosis [22]. Another study by Aktar et al. reported that NLR may reach abnormally high levels in children with brucella arthritis [5]. In our study, there were no significant differences in the median WBC, NEU, LYM, NLR, and PLT values between the brucellosis positive and negative groups, whereas the median AST, ALT, GGT, CRP, and ESR values were significantly higher in the positive group. Similarly, none of the WBC, NEU, LYM, NLR, and PLT values were found to be significant in the ROC analysis ( $p>0.05$ ) (Table 4). These results suggested that WBC, NEU, LYM, NLR, and PLT values might not always be used as supportive parameters in the differential diagnosis of childhood brucellosis. Although the ROC analysis yielded significant results for the AST, ALT, GGT, CRP, and ESR levels; the specificity and sensitivity of these parameters did not reach adequate levels ( $p<0.05$ ) (Table 4). The ROC analysis revealed significant results for an ESR cut-off value of $15.5 \mathrm{~mm} / \mathrm{g}$ with $68.3 \%$ sensitivity and $62.9 \%$ specificity. These results suggest that high levels of AST, ALT, GGT, CRP, and ESR can be used as adjunct parameters in the differential diagnosis of childhood brucellosis. However, the role of these markers in the differential diagnosis is limited due to their low specificity and sensitivity. 
The major limitation of this study is that the control group did not consist of healthy children. We thought that this limitation might have contributed to the absence of a significant difference in the complete blood count parameters between the brucellosis positive and negative groups. Also, we think that the low number of blood culture-positive cases might have contributed to the low specificity and sensitivity found for the inflammation markers in ROC analysis.

\section{Conclusions}

In developing countries where consumption of unpasteurized milk and dairy products is widespread, brucellosis is common especially in boys born to families engaged in animal husbandry in rural areas. A potential diagnosis of brucellosis should be remembered in the presence of joint involvement, prolonged fever, and organomegaly. Despite the insufficient levels of specificity and sensitivity, we are of the opinion that high levels of AST, ALT, GGT, CRP, and ESR may have a complementary role in the differential diagnosis of childhood brucellosis.

\section{Additional Information \\ Disclosures}

Human subjects: Consent was obtained by all participants in this study. Harran University, Medical Faculty Ethics Committee issued approval 19747. This study conformed to the principles of the 2008 Declaration of Helsinki and was approved by the local ethics committee of Harran University, Medical Faculty, Turkey (Approval date and number: 03.05.2018, Session 5, 19747). Animal subjects: All authors have confirmed that this study did not involve animal subjects or tissue. Conflicts of interest: In compliance with the ICMJE uniform disclosure form, all authors declare the following: Payment/services info: All authors have declared that no financial support was received from any organization for the submitted work. Financial relationships: All authors have declared that they have no financial relationships at present or within the previous three years with any organizations that might have an interest in the submitted work. Other relationships: All authors have declared that there are no other relationships or activities that could appear to have influenced the submitted work.

\section{Acknowledgements}

The authors would like to thank all employees in the Şanlıurfa Training and Research Hospital and Harran University Faculty of Medicine Department of Pediatrics Clinic for their valuable contribution.

\section{References}

1. Keşli R, Bilgin H, Yılmaz H: Determination of in vitro susceptibilities of Brucella spp. strains against 11 different antibacterial gents isolated from blood cultures. Mikrobiyol Bul. 2017, 51:260-268. 10.5578/mb.57362

2. Ning C, Shuyi G, Tao Y, Hao Z, Zhang X. : Epidemiological survey of human brucellosis in Inner Mongolia, China, 2010-2014: A high risk groups-based survey. J Infect Public Health. 2018, 11:24-29. 10.1016/j.jiph.2017.02.013

3. Çiftdoğan DY, Aslan S: Unrecognized pediatric and adult family members of children with acute brucellosis . Braz J Infect Dis. 2017, 21:520-524. 10.1016/j.bjid.2017.05.006

4. Njeru J, Wareth G, Melzer F, Henning K, Pletz MW, Heller R, Neubauer H.: Systematic review of brucellosis in Kenya: disease frequency in humans and animals and risk factors for human infection. BMC Public Health. 2016, 16:853. 10.1186/s12889-016-3532-9

5. Aktar F, Tekin R, Bektaş MS, et al.: Diagnostic role of inflammatory markers in pediatric Brucella arthritis . Ital J Pediatr. 2016, 42:3. 10.1186/s13052-016-0211-5

6. Kitt E, Brannock KR, VonHolz LA, Planet PJ, Graf E, Pillai V. : A case report of pediatric brucellosis in an Algerian immigrant. Open Forum Infect Dis. 2017, 4:263. 10.1093/ofid/ofw263

7. Wang X, Yan Y, Wu F, et al.: Sixteen Chinese pediatric brucellosis patients onset of fever in non-epidemic areas. and 8 developed with osteoarticular involvement. Clin Rheumatol. 2018, 37:145-149. 10.1007/s10067-017-3819-y

8. Bilici M, Demir F, Yllmazer MM, Bozkurt F, Tuzcu V. : Brucella infection associated with complete atrioventricular block. Balkan Med J. 2016, 33:556-558. 10.5152/balkanmedj.2016.140684

9. Çetin M, Turfan N, Karaman K, Yaşar AŞ, Güven B, Tunçdemir P. : The pattern of Tpeak-Tend interval and QTdis, and Pdis in children with brucellosis. J Trop Pediatr. 2019, 65:474-480. 10.1093/tropej/fmy078

10. Dal T, Kara SS, Cikman A, et al.: Comparison of multiplex real-time polymerase chain reaction with serological tests and culture for diagnosing human brucellosis. J Infect Public Health. 2019, 12:337-342. 10.1016/j.jiph.2018.11.008

11. Torkaman Asadi F, Hashemi SH, Alikhani MY, Moghimbeigi A, Naseri Z. : Clinical and diagnostic aspects of brucellosis and antimicrobial susceptibility of brucella isolates in Hamedan, Iran. Jpn J Infect Dis. 2017, 24:235-238. 10.7883/yoken.JJID.2016.133

12. Ulu-Kilic A, Metan G, Alp E: Clinical presentations and diagnosis of brucellosis. Recent Pat Antiinfect Drug Discov. 2013, 8:34-41. 10.2174/1574891X11308010007

13. Koyuncu I, Kocyigit A, Ozer A, Selek S, Kirmit A, Karsen H. : Diagnostic potential of Brucella melitensis Rev1 native Omp28 precursor in human brucellosis. Cent Eur J Immunol. 2018, 43:81-89. 10.5114/ceji.2018.74877

14. Díaz R, Casanova A, Ariza J, Moriyón I. : The Rose Bengal Test in human brucellosis: a neglected test for the diagnosis of a neglected disease. PLoS Negl Trop Dis. 2011, 5:950. 10.1371/journal.pntd.0000950 
15. Gül S, Satilmiş OK, Ozturk B, Gökçe MI, Kuscu F. : Seroprevalence of brucellosis among children in the Middle Anatolia Region of Turkey. J Health Popul Nutr. 2014, 32:577-579.

16. Çıraklı S, Karlı A, Şensoy G, Belet N, Yanık K, Çıraklı A. : Evaluation of childhood brucellosis in the central Black Sea region. Turk J Pediatr. 2015, 57:123-128.

17. Dutta D, Sen A, Gupta D, Kuila P, Chatterjee D, Sanyal S, Das S.: Childhood brucellosis in eastern India . Indian J Pediatr. 2018, 85:266-271. 10.1007/s12098-017-2513-Z

18. Sutcu M, Gokceer D, Akturk H, et al.: Brucella infection in a child with progressive familial intrahepatic cholestasis type 2 who had undergone liver transplantation. Pediatr Transplant. 2015, 19:146-148. 10.1111/petr.12555

19. Aypak A, Aypak C, Bayram Y: Hematological findings in children with brucellosis . Pediatr Int. 2015, 57:1108-1111. 10.1111/ped.12688

20. Pourakbari B, Abdolsalehi M, Mahmoudi S, Banar M, Masoumpour F, Mamishi S. : Epidemiologic, clinical, and laboratory characteristics of childhood brucellosis :A study in an Iranian children's referral hospital. Wien Med Wochenschr. 2019, 169:232-239. 10.1007/s10354-019-0685-z

21. Fanni F, Shahbaznejad L, Pourakbari B, Mahmoudi S, Mamishi S. : Clinical manifestations, laboratory findings, and therapeutic regimen in hospitalized children with brucellosis in an Iranian Referral Children Medical Centre. J Health Popul Nutr. 2013, 31:218-222.

22. Bozdemir ŞE, Altıntop YA, Uytun S, Aslaner H, Torun YA. : Diagnostic role of mean platelet volume and neutrophil to lymphocyte ratio in childhood brucellosis. Korean J Intern Med. 2017, 32:1075-1081. 10.3904/kjim.2016.092

23. Kara SS, Cayir Y: Predictors of blood culture positivity in pediatric brucellosis . J Coll Physicians Surg Pak. 2019, 29:665-670. 10.29271/jcpsp.2019.07.665

24. He J, Zhang Q: Hip osteoarticular complication due to delay in diagnosis and treatment of brucellar hip arthritis: two cases report. BMC Infect Dis. 2019, 19:412. 10.1186/s12879-019-4045-9

25. Arapović J, Špičić S, Ostojić M, Duvnjak S, Arapović M, Nikolić J, Cvetnić Ž. : Epidemiological, clinical, and molecular characterization of human brucellosis in Bosnia and Herzegovina - an ongoing brucellosis outbreak. Acta Med Acad. 2018, 47:50-60. 10.5644/ama2006-124.214

26. Sahinturk H, Baran B, Sisman G, Altun R: Liver involvement is associated with blood culture positivity and high agglutination titre in patients with brucellosis in Turkey. J Med Microbiol. 2018, 67:1078-1082. 10.1099/jmm.0.000791

27. Apa H, Devrim I, Memur S, et al.: Factors affecting Brucella spp. blood cultures positivity in children . Vector Borne Zoonotic Dis. 2013, 13:176-180. 10.1089/vbz.2012.0997 